\title{
Green $\mathrm{CsSnX}_{3}(\mathrm{X}=\mathrm{Cl}, \mathrm{Br}, \mathrm{I})$ - Derived Quantum Dots For Photovoltaic Applications: First Principles Investigations
}

Bhakti Khirsagar,, ฯ Namrata Jaykhedkar, ${ }^{\ddagger} @$ Kalpna Jain, ${ }^{\S}$ Shyam Kishor,॥

Vaishali Shah, ${ }^{*, \ddagger}$, Lavanya M. Ramaniah ${ }^{*, \perp, \#}$ and Shivani Tiwari, ${ }^{\ddagger}$

${ }^{\ddagger}$ Department of Scientific Computing, Modeling and Simulation, Savitribai Phule Pune University, Pune, India.

『 Department of Physics, Savitribai Phule Pune University, Pune, India.

$\S$ Department of Physics, D. J. College, Baraut 250611, Uttar Pradesh, India.

"Department of Chemistry, J. V. College, Baraut 250611, Uttar Pradesh, India.

${ }^{\perp}$ High Pressure Physics Division, Bhabha Atomic Research Centre, Trombay, Mumbai, India.

\# Homi Bhabha National Institute, Mumbai, India.

${ }^{\circledR}$ Laboratory for Advanced Materials, Faculty of Natural Sciences, Comenius University, 84104, Bratislava, Slovakia.

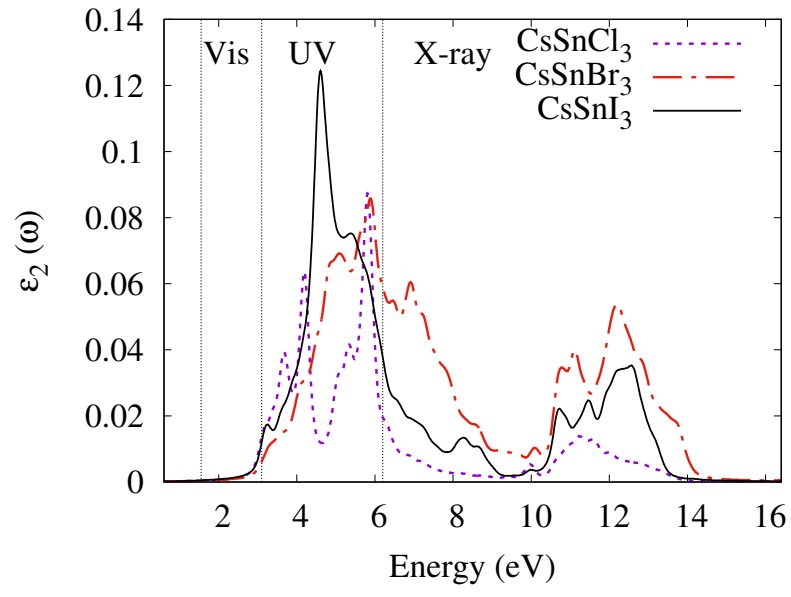

(a)

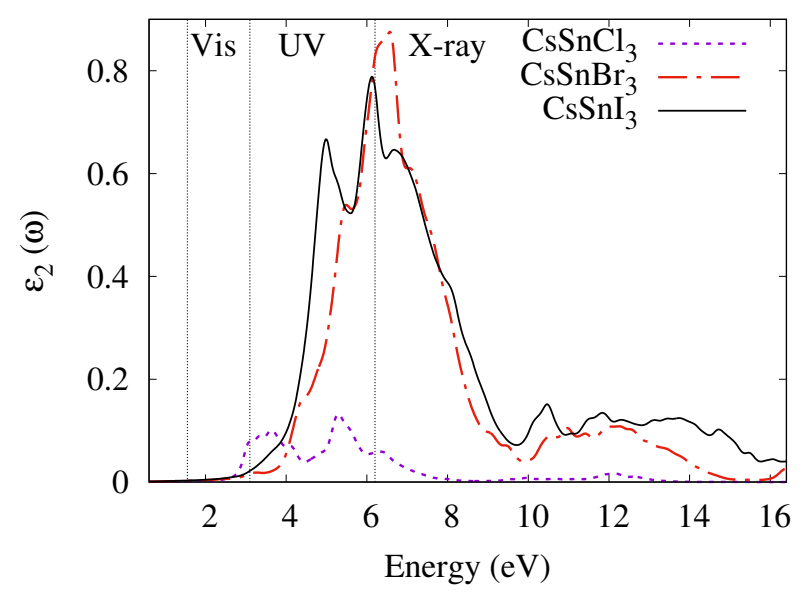

(b)

Figure S1: Absorption spectra calculated using DFPT[1] for (a) QD-1 with $\mathrm{OH}^{-}$and (b) QD-2 with $\mathrm{H}^{+}$ligation. $\mathrm{CsSnCl}_{3}$ QDs show weakest absorption in the entire energy range. 


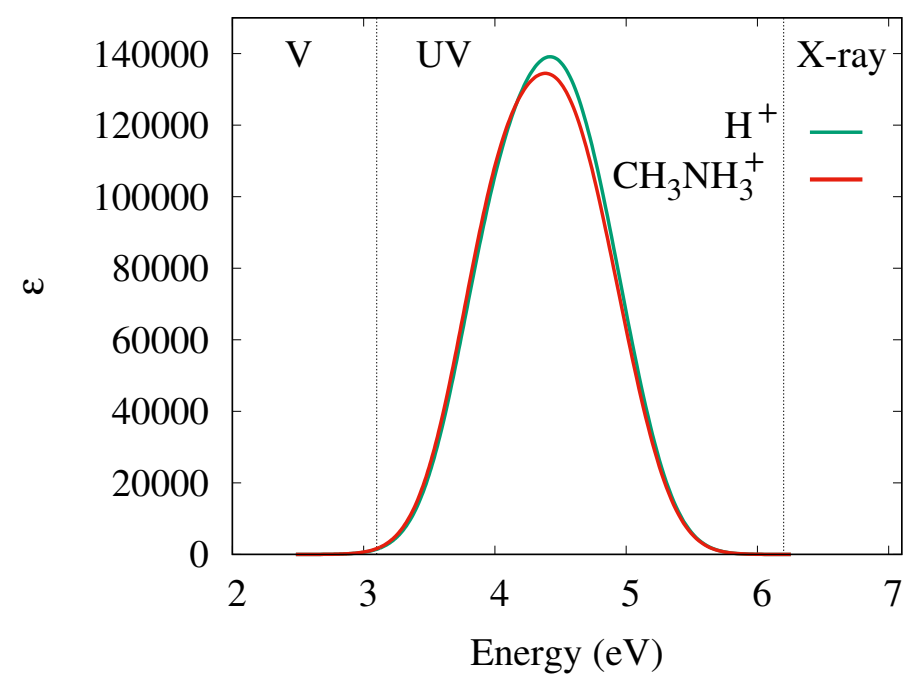

Figure S2: Absorption spectra from TDDFT [2] for QD-2 with $\mathrm{H}^{+}$and $\mathrm{CH}_{3} \mathrm{NH}_{3}^{+}$ligation. The absorption spectra span the ultraviolet region of the electromagnetic spectrum.

Table S1: Optical excitation data of the QDs calculated using TDDFT. $\mathrm{S}_{0}$ denotes the ground state of the QD. $\mathrm{E}_{e x}$ is the optical excitation energy associated with the absorbed wavelength $\lambda$ for the electronic transition to the excited state $\mathrm{S}_{N}$. The oscillator strength $f$ associated with the transition enables comparison of relative probabilities for absorption.

\begin{tabular}{llllll}
\hline Quantum dot & $\begin{array}{l}\text { Excited states } \\
\left(@ \mathrm{~S}_{0}\right)\end{array}$ & $\begin{array}{l}\mathrm{E}_{\text {ex }} \\
(\mathrm{eV})\end{array}$ & $\begin{array}{l}\lambda \\
(\mathrm{nm})\end{array}$ & $\begin{array}{l}\text { Oscillator } \\
\text { strength }(f)\end{array}$ & $\begin{array}{l}\text { Eigenvalues } \\
(\mathrm{NTO})\end{array}$ \\
\hline CsSnCl $_{3}$ & & & & & \\
QD-1 & & & & & \\
& $\mathrm{S}_{0} \rightarrow \mathrm{S}_{6}$ & 2.40 & 515.81 & 0.0502 & 0.584 \\
& $\mathrm{~S}_{0} \rightarrow \mathrm{S}_{10}$ & 2.47 & 501.74 & 0.1348 & 0.628 \\
& $\mathrm{~S}_{0} \rightarrow \mathrm{S}_{18}$ & 2.63 & 470.97 & 0.0484 & 0.534 \\
& $\mathrm{~S}_{0} \rightarrow \mathrm{S}_{86}$ & 3.40 & 364.03 & 0.0141 & 0.300 \\
& $\mathrm{~S}_{0} \rightarrow \mathrm{S}_{89}$ & 3.42 & 362.25 & 0.0211 & 0.531 \\
& $\mathrm{~S}_{0} \rightarrow \mathrm{S}_{109}$ & 3.62 & 342.65 & 0.0159 & 0.511 \\
& $\mathrm{~S}_{0} \rightarrow \mathrm{S}_{121}$ & 3.71 & 332.70 & 0.0152 & 0.626 \\
QD-2 & & & & & \\
& $\mathrm{S}_{0} \rightarrow \mathrm{S}_{8}$ & 3.91 & 317.17 & 0.3231 & 0.683 \\
& $\mathrm{~S}_{0} \rightarrow \mathrm{S}_{9}$ & 3.92 & 316.25 & 0.3862 & 0.656 \\
& & & & & \\
& $\mathrm{~S}_{0} \rightarrow \mathrm{S}_{9}$ & 3.93 & 314.92 & 0.2685 & 0.557 \\
& $\mathrm{~S}_{0} \rightarrow \mathrm{S}_{18}$ & 4.09 & 303.22 & 0.2049 & 0.407
\end{tabular}

$\mathrm{CsSnBr}_{3}$

$\begin{array}{llllll}\text { QD-1 } & \mathrm{S}_{0} \rightarrow \mathrm{S}_{6} & 2.44 & 509.07 & 0.0505 & 0.566 \\ & \mathrm{~S}_{0} \rightarrow \mathrm{S}_{10} & 2.50 & 496.81 & 0.1237 & 0.542 \\ & \mathrm{~S}_{0} \rightarrow \mathrm{S}_{18} & 2.64 & 468.85 & 0.0265 & 0.433 \\ & \mathrm{~S}_{0} \rightarrow \mathrm{S}_{91} & 3.44 & 359.59 & 0.0162 & 0.471 \\ & \mathrm{~S}_{0} \rightarrow \mathrm{S}_{136} & 3.85 & 321.99 & 0.0129 & 0.316 \\ & \mathrm{~S}_{0} \rightarrow \mathrm{S}_{145} & 3.89 & 318.67 & 0.0212 & 0.361 \\ & & & & & \\ \text { QD-2 } & \mathrm{S}_{0} \rightarrow \mathrm{S}_{8} & 3.54 & 350.29 & 0.2755 & 0.645 \\ & \mathrm{~S}_{0} \rightarrow \mathrm{S}_{10} & 3.56 & 348.24 & 0.3862 & 0.692\end{array}$


$\mathrm{CsSnI}_{3}$

$\begin{array}{clllll}\text { QD-1 } & \mathrm{S}_{0} \rightarrow \mathrm{S}_{20} & 2.48 & 498.85 & 0.0291 & 0.472 \\ & \mathrm{~S}_{0} \rightarrow \mathrm{S}_{22} & 2.50 & 496.15 & 0.0386 & 0.378 \\ \mathrm{~S}_{0} \rightarrow \mathrm{S}_{24} & 2.52 & 491.01 & 0.0632 & 0.424 \\ \mathrm{~S}_{0} \rightarrow \mathrm{S}_{38} & 2.68 & 460.95 & 0.0169 & 0.512 \\ & \mathrm{~S}_{0} \rightarrow \mathrm{S}_{42} & 2.73 & 453.34 & 0.0501 & 0.450 \\ & \mathrm{~S}_{0} \rightarrow \mathrm{S}_{52} & 2.93 & 422.63 & 0.0168 & 0.626 \\ & \mathrm{~S}_{0} \rightarrow \mathrm{S}_{115} & 3.70 & 334.36 & 0.0403 & 0.532 \\ & \mathrm{~S}_{0} \rightarrow \mathrm{S}_{119} & 3.72 & 332.75 & 0.0847 & 0.528 \\ & \mathrm{~S}_{0} \rightarrow \mathrm{S}_{125} & 3.77 & 328.36 & 0.0762 & 0.641\end{array}$

Near $3 \mathrm{eV}$

\begin{tabular}{llllll} 
QD-1 & $\mathrm{S}_{0} \rightarrow \mathrm{S}_{58}$ & 2.99 & 413.56 & 0.0114 & 0.378 \\
& $\mathrm{~S}_{0} \rightarrow \mathrm{S}_{60}$ & 3.01 & 411.42 & 0.0022 & 0.424 \\
& $\mathrm{~S}_{0} \rightarrow \mathrm{S}_{61}$ & 3.06 & 404.41 & 0.0010 & 0.464 \\
& $\mathrm{~S}_{0} \rightarrow \mathrm{S}_{62}$ & 3.07 & 404.28 & 0.0016 & 0.454 \\
& $\mathrm{~S}_{0} \rightarrow \mathrm{S}_{65}$ & 3.12 & 397.77 & 0.0062 & 0.350 \\
& $\mathrm{~S}_{0} \rightarrow \mathrm{S}_{66}$ & 3.12 & 397.66 & 0.0041 & 0.355 \\
& $\mathrm{~S}_{0} \rightarrow \mathrm{S}_{68}$ & 3.14 & 394.16 & 0.0018 & 0.407 \\
& $\mathrm{~S}_{0} \rightarrow \mathrm{S}_{69}$ & 3.15 & 393.68 & 0.0013 & 0.495 \\
& $\mathrm{~S}_{0} \rightarrow \mathrm{S}_{71}$ & 3.15 & 392.92 & 0.0027 & 0.445 \\
& & & & & \\
& $\mathrm{~S}_{0} \rightarrow \mathrm{S}_{1}$ & 2.80 & 443.04 & 0.0359 & 0.678 \\
& $\mathrm{~S}_{0} \rightarrow \mathrm{S}_{4}$ & 3.01 & 412.35 & 0.0350 & 0.510 \\
& $\mathrm{~S}_{0} \rightarrow \mathrm{S}_{7}$ & 3.10 & 400.23 & 0.0524 & 0.668 \\
& $\mathrm{~S}_{0} \rightarrow \mathrm{S}_{8}$ & 3.14 & 395.39 & 0.2806 & 0.659 \\
& $\mathrm{~S}_{0} \rightarrow \mathrm{S}_{9}$ & 3.16 & 391.65 & 0.2139 & 0.415 \\
& $\mathrm{~S}_{0} \rightarrow \mathrm{S}_{10}$ & 3.17 & 390.67 & 0.0917 & 0.509 \\
& $\mathrm{~S}_{0} \rightarrow \mathrm{S}_{11}$ & 3.18 & 389.44 & 0.0798 & 0.444 \\
& $\mathrm{~S}_{0} \rightarrow \mathrm{S}_{14}$ & 3.21 & 386.31 & 0.0873 & 0.317 \\
\hline
\end{tabular}




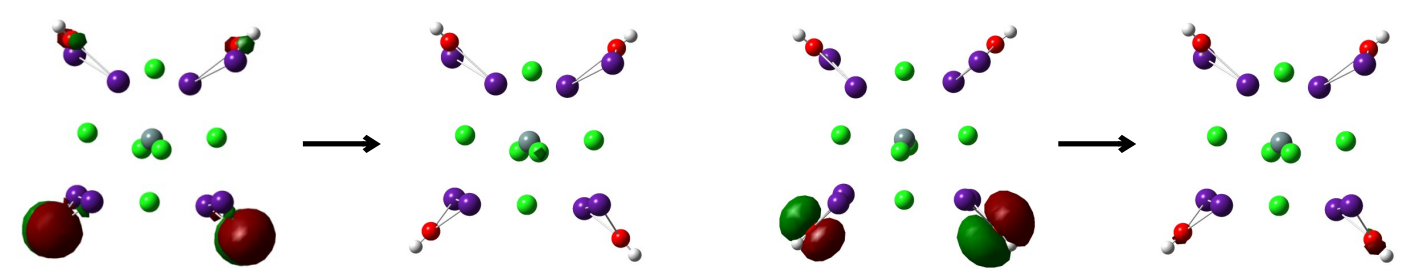

ES-6: $E=2.40, f=0.0502$

ES-86: $E=3.40, f=0.0141$

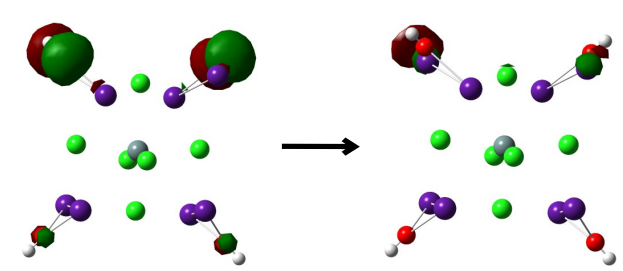

ES-10: $E=2.47, f=0.1348$

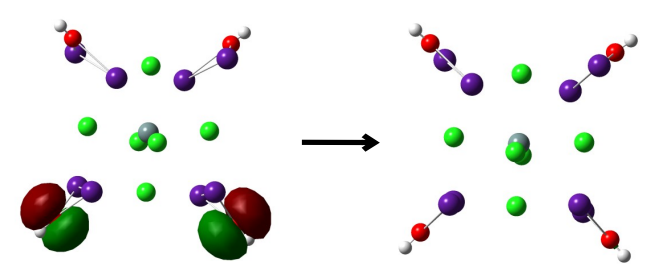

ES-89: $E=3.42, f=0.0211$

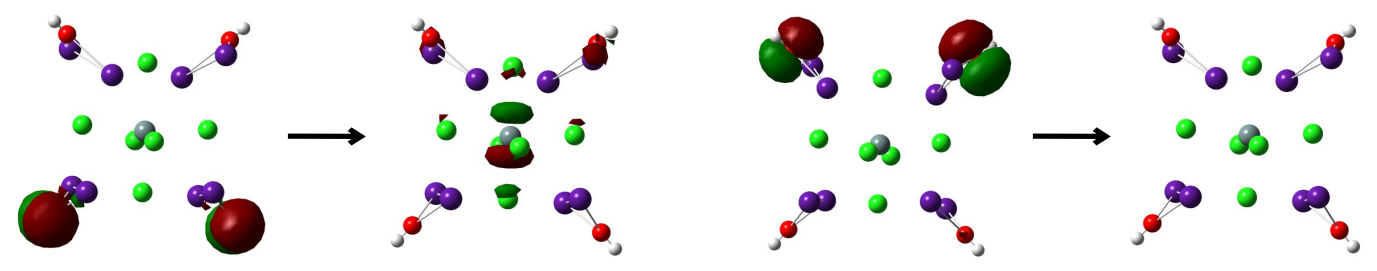

ES-18: $\mathrm{E}=2.63, \mathrm{f}=0.0484$

ES-109: $E=3.62, f=0.0159$

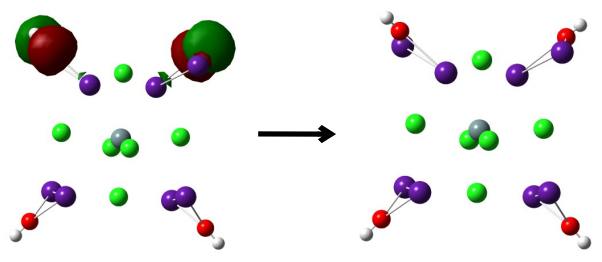

ES-121: $E=3.71, f=0.0152$

Figure S3: Natural transition orbitals (NTOs) for the transitions (mentioned in the figures) in $\mathrm{CsSnCl}_{3}$ QD-1. 


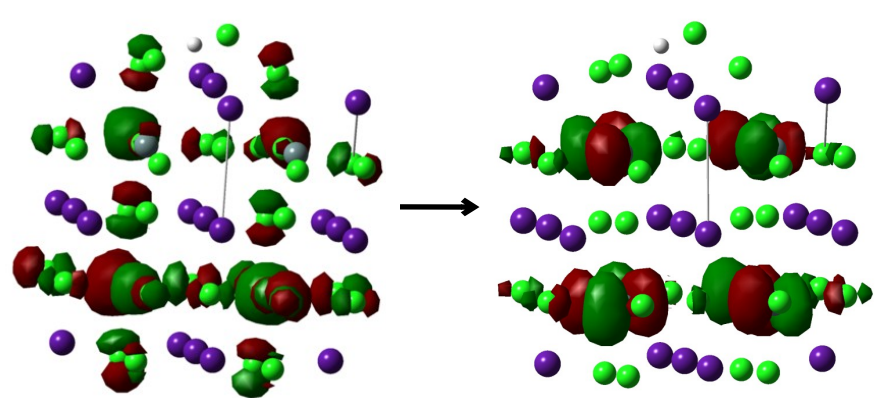

ES-8: $E=3.91, f=0.3231$

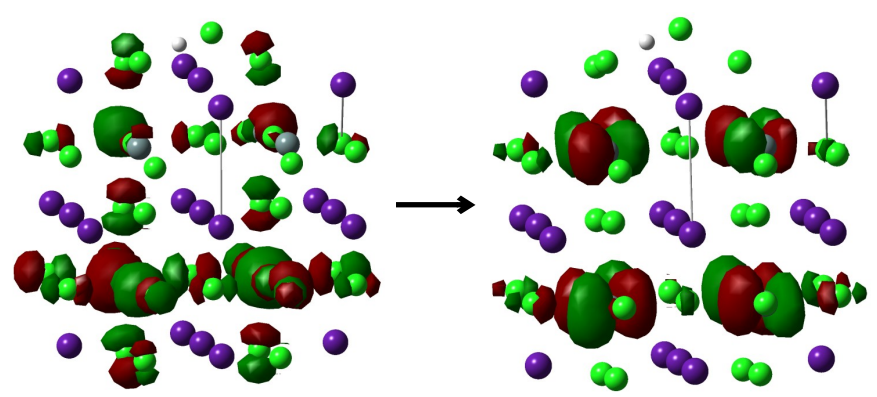

ES-9: $\mathrm{E}=3.92, \mathrm{f}=0.3862$

Figure S4: Natural transition orbitals (NTOs) for the transitions (mentioned in the figures) in $\mathrm{CsSnCl}_{3} \mathrm{QD}-2$.

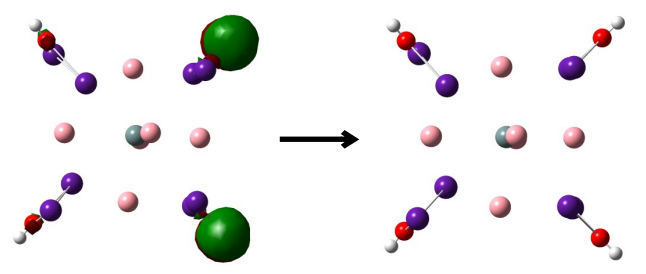

ES-6: $E=2.44, f=0.0505$

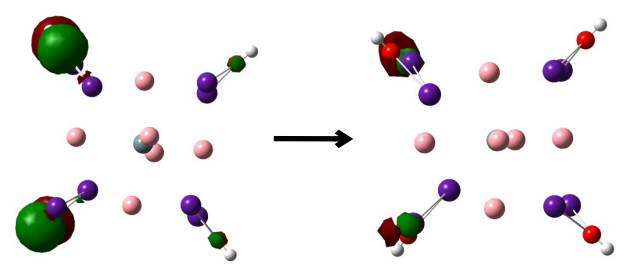

ES-10: $E=2.50, f=0.1237$

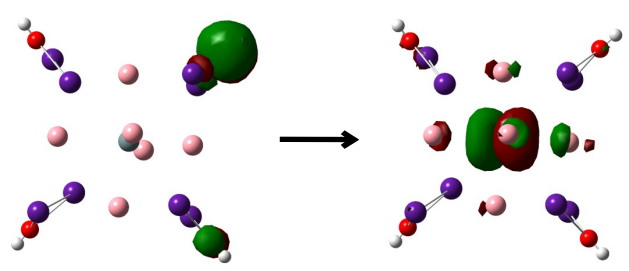

ES-18: $E=2.64, f=0.0265$

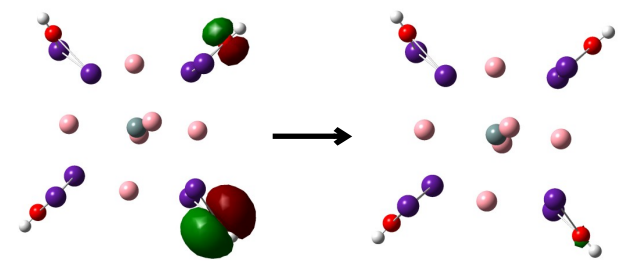

ES-91: $\mathrm{E}=3.44, \mathrm{f}=0.0162$

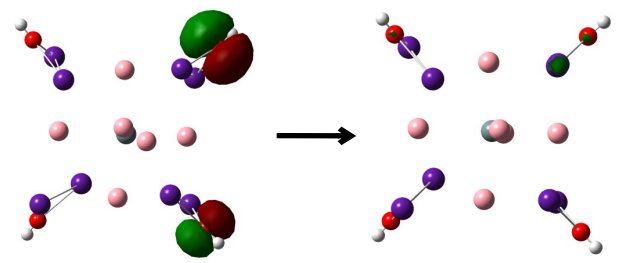

ES-136: $E=3.85, f=0.0129$

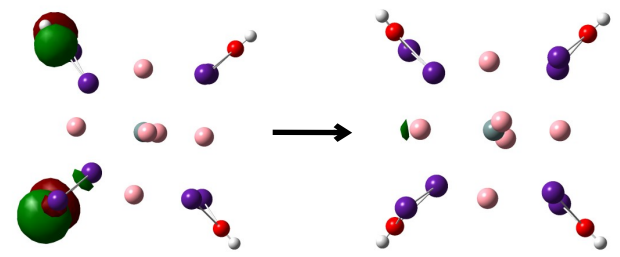

ES-145: $E=3.89, f=0.0212$

Figure S5: Natural transition orbitals (NTOs) for the transitions (mentioned in the figures) in $\mathrm{CsSnBr}_{3} \mathrm{QD}-1$ 


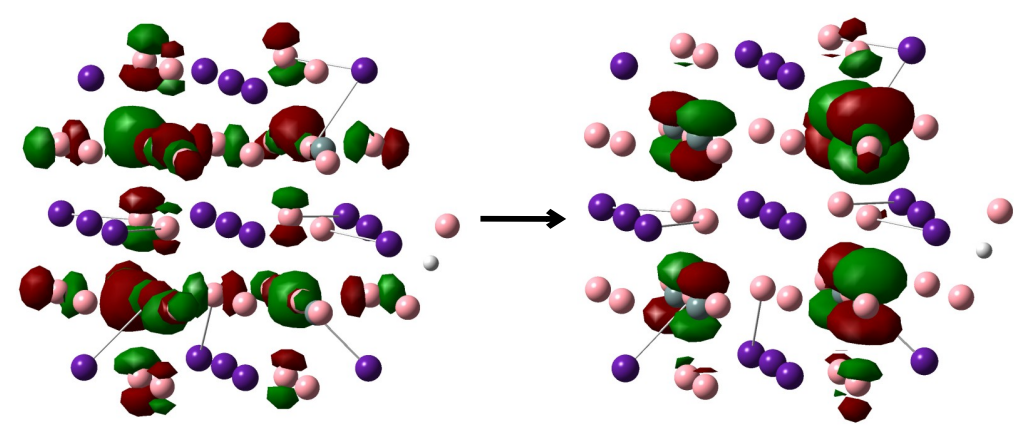

ES-8: $E=3.54, f=0.2755$

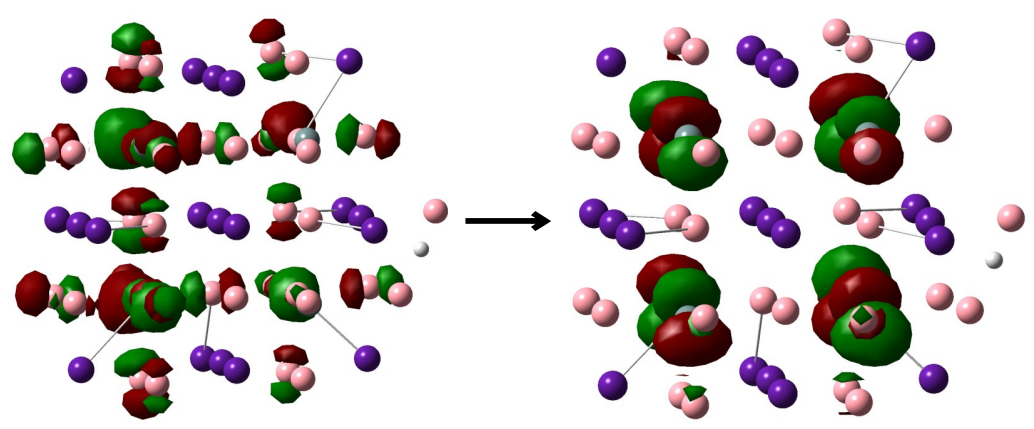

ES-10: $\mathrm{E}=3.56, \mathrm{f}=0.3862$

Figure S6: Natural transition orbitals (NTOs) for the transitions (mentioned in the figures) in $\mathrm{CsSnBr}_{3}$ QD-2.

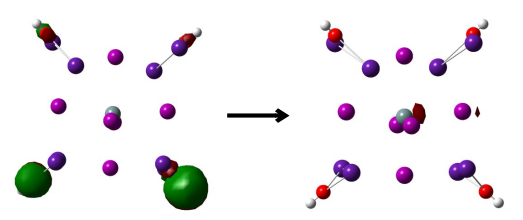

ES-20: $E=2.48, f=0.0291$

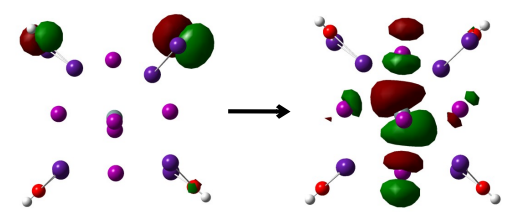

ES-22: $E=2.50, f=0.0386$

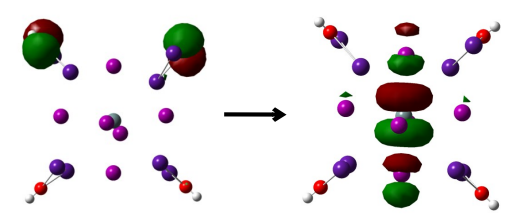

ES-24: $E=2.52, f=0.0632$

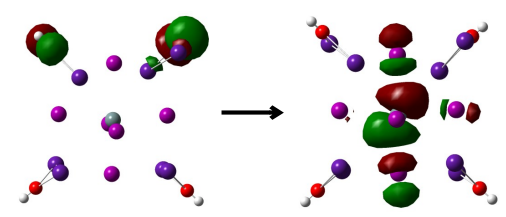

ES-38: $E=2.68, f=0.0169$

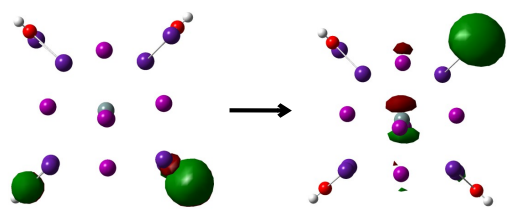

$\mathrm{ES}-42: \mathrm{E}=2.73, \mathrm{f}=0.0501$

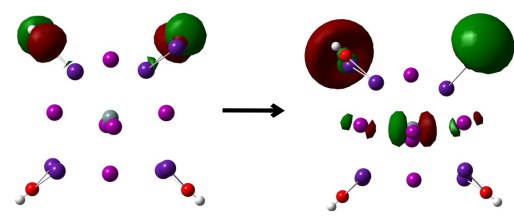

ES-52: $E=2.93, f=0.0168$

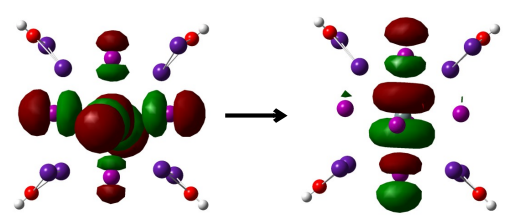

ES-115: $E=3.70, f=0.0403$

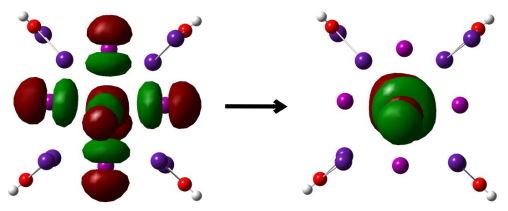

ES-119: $E=3.72, f=0.0847$

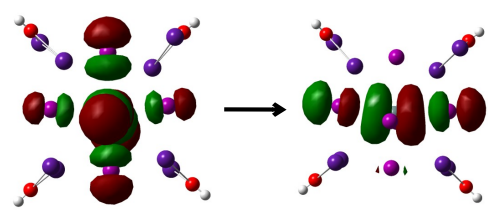

ES-125: $E=3.77, f=0.0762$

Figure S7: Natural transition orbitals (NTOs) for the transitions (mentioned in the figures) in $\mathrm{CsSnI}_{3} \mathrm{QD}-1$. 


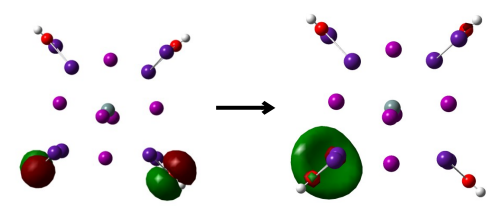

ES-58: $\mathrm{E}=2.99, \mathrm{f}=0.0114$

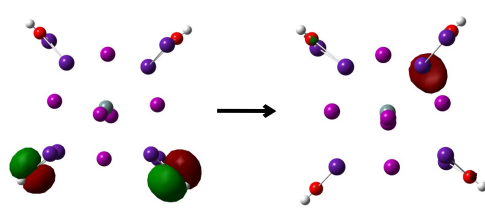

ES-60: $E=3.0136, f=0.0022$

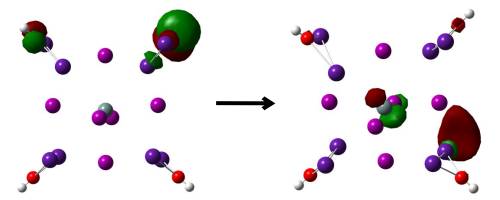

ES-61: $E=3.06, f=0.0010$

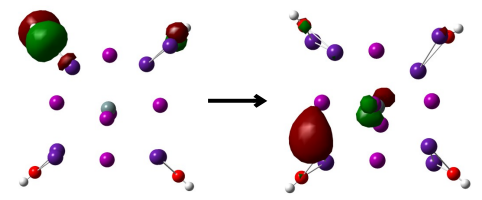

ES-62: $E=3.07, f=0.0016$

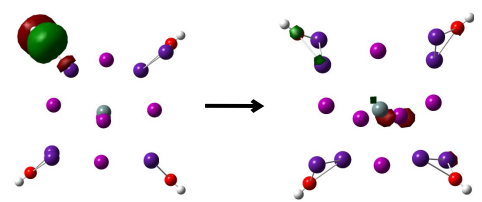

ES-65: $E=3.12, f=0.0062$

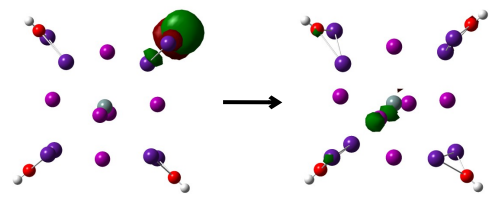

ES-66: $E=3.12, f=0.0041$

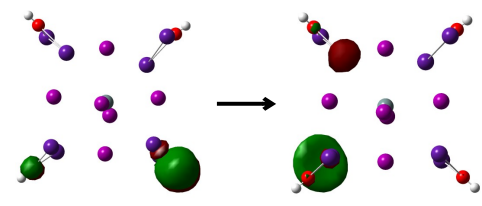

ES-68: $E=3.14, f=0.0018$

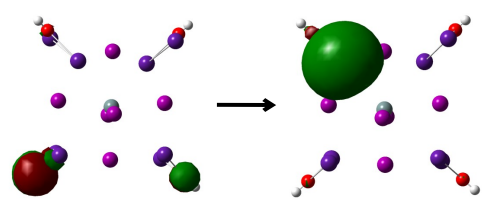

ES-69: $E=3.15, f=0.0013$

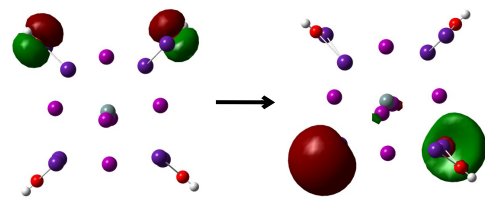

ES-71: $\mathrm{E}=3.15, \mathrm{f}=0.0027$

Figure S8: Natural transition orbitals (NTOs) for the transitions (mentioned in the figures) in $\mathrm{CsSnI}_{3} \mathrm{QD}-1 \approx 3 \mathrm{eV}$. 


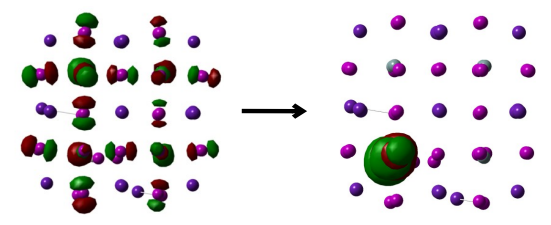

$\mathrm{ES}-1: \mathrm{E}=2.80, \mathrm{f}=0.0359$

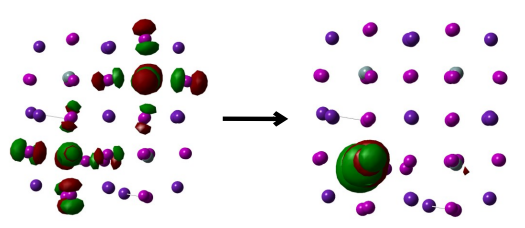

ES-4: $E=3.01, f=0.0350$

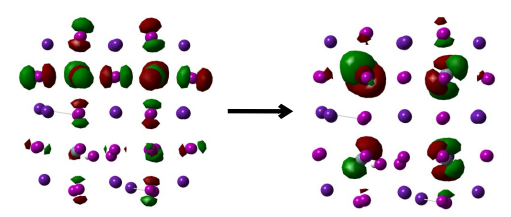

$E S-7: E=3.10, f=0.0524$

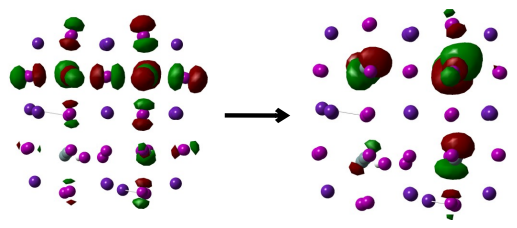

ES-8: $E=3.14, f=0.2806$

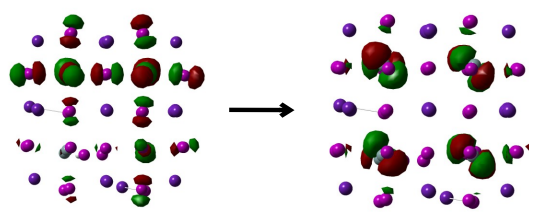

ES-9: $\mathrm{E}=3.16, \mathrm{f}=0.2139$

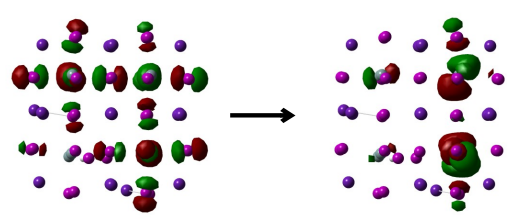

ES-10: $E=3.17, f=0.0917$

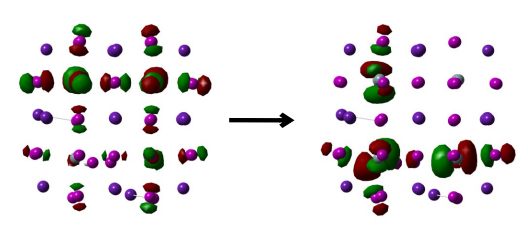

ES-11: $E=3.18, f=0.0798$

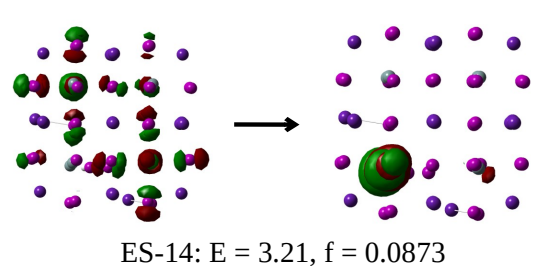

ES-14: $E=3.21, f=0.0873$

Figure S9: Natural transition orbitals (NTOs) for the transitions (mentioned in the figures) in $\mathrm{CsSnI}_{3} \mathrm{QD}-2$.

\section{References}

[1] Kresse, G.; Furthmüller, J. Efficient iterative schemes for ab initio total-energy calculations using a plane-wave basis set. Phys. Rev. B 1996, 54, 11169-11186.

[2] Frisch, M. J. et al. Gaussian 16 Revision B.01. 2016; Gaussian Inc. Wallingford CT. 